\title{
Rare cases of pulmonary inflammatory myofibroblastic tumors in adult male patients: a case report
}

\author{
Hui Li", Yanfa He", Bin Wang, Xinliang Song, Dawei Zhao, Hang Yu, Kelei Qi, Tao Liu \\ Department of Thoracic Surgery, Hebei Provincial Chest Hospital, Shijiazhuang, China \\ \#These authors contributed equally to this work. \\ Correspondence to: Tao Liu. Department of Chest Surgery, Hebei Provincial Chest Hospital, Shijiazhuang 050000, China. Email: spielberg0923@163.com.
}

\begin{abstract}
Pulmonary inflammatory myofibroblastic tumors (IMTs) are rarely reported in adult males. Given the low incidence of IMT and the lack of imaging references and pathological guidance, the misdiagnosis rate of IMT is high. In this article, we describe two cases of IMTs in the lungs. Both patients were adult males with lesions in the right lobe, a history of pulmonary tuberculosis, and a long period of refractory intermittent pulmonary inflammation. Our two male patients both experienced intermittent cough symptoms, but pulmonary IMTs were not suspected for a long time. Both patients were diagnosed with pulmonary tuberculosis before IMT was confirmed and treated with isoniazid (H), rifampin (R), pyrazinamide $(\mathrm{Z})$, and ethambutol (E) (HRZE) or isoniazid (H), levofloxacin (L), pyrazinamide (Z), and ethambutol (E) (HLZE) for months. In Case 2, we observed multiple subpleural cord signs in the left lung, soft tissue mass shadows at the apex of the right upper lobe, a thickened interlobular interval, and scattered patches and nodules in the upper right lung. These features are novel in the identification of IMTs. Both of the pathological findings revealed a great deal of myofibroblasts, fibroblasts and collagen fibers in the lower right lung lesion, accompanied by a large number of plasma cells and foam cell infiltration, which were consistent with the features of IMT. The two patients displayed exceedingly different symptoms, computed tomography (CT) imaging features, and pathological results from those reported in traditional records. These findings provide novel references that will extend understandings of this rare disease.
\end{abstract}

Keywords: Inflammatory myofibroblastic tumor (IMT); lung cancer; case report; inflammatory pseudotumor

Submitted Jul 15, 2021. Accepted for publication Sep 10, 2021.

doi: $10.21037 /$ tcr-21-1683

View this article at: https://dx.doi.org/10.21037/tcr-21-1683

\section{Introduction}

An inflammatory myofibroblastic tumor (IMT) is a rare type of intermediate tumor that often affects children and young adults (it is especially common in children younger than 16 years old). Knowledge of IMT is limited; however, it is usually considered a type of benign spindle cell tumor with malignant potential. In 2020, the World Health Organization (WHO) defined IMT as a unique metastatic tumor, composed of myofibroblastic spindle cells, accompanied by an inflammatory infiltrate of chronic inflammatory cells, such as fibroblasts, plasma cells, lymphocytes, or/and eosinophils. IMT can occur throughout the body; however, the most frequent sites are the viscera and soft tissues, including lung, peritoneum and mesentery (1-3). Additionally, IMT occasionally occurs in the trachea, stomach, and small intestine (4-6). The firstchoice treatment for IMT (especially pulmonary IMT) is a R0 resection, preferably with video-assisted thoracoscopic surgery $(7,8)$. Given the low incidence of IMT, and the lack of imaging references and pathological guidance, the misdiagnosis rate of IMT is high.

IMT occurs extremely rare in male adults and is rarely reported in the lung. In this article, we present two extremely rare cases of patients with pulmonary IMTs. Both patients were male adults, who had long misdiagnosis histories, and shared some common symptoms. These findings provide novel references that will extend 
understandings of the onset of pulmonary IMT. We also provide details of the CT imaging and pathological features of IMT.

We present the following article in accordance with the CARE reporting checklist (available at https://dx.doi. org/10.21037/tcr-21-1683).

\section{Case presentation}

All procedures performed in studies involving human participants were in accordance with the ethical standards of the institutional and/or national research committee(s) and with the Helsinki Declaration (as revised in 2013). Written informed consent was obtained from the patient for publication of this case report and accompanying images. A copy of the written consent is available for review by the editorial office of this journal.

\section{Case 1}

A 25-year-old male patient was admitted to our hospital on February 1, 2020, complaining of an intermittent cough, which he had experienced for half a year. During this time, no obvious irritating dry cough, sputum, chest tightness, shortness of breath, or chest pain was noticed. The intermittent treatment of the cough did not have a satisfactory effect. One month earlier, a mass was found in the patient's right lung. In 2013, the patient had been diagnosed with left pulmonary tuberculosis and left side tuberculous pleurisy, and treated with isoniazid, rifampicin, pyrazinamide, ethambutol, and levofloxacin for 4 months. An enhanced computed tomography (CT) examination was performed on February 11, 2020. The results showed a 5.6-cm mass in the patient's lower lobe of the right lung with a smooth edge and a clear tumor-lung interface. The patient was previously healthy, normotensive, did not have a history of alcohol intake or smoking.

On February 13, 2020, a pulmonary puncture smear and puncture pathological examination were performed. The results of the smear showed heterogeneous cells, and the pathological examination of the puncture tissue showed inflammatory hyperplasia and coagulation necrosis. In the pathological consultation of the sampled tissue sections, necrosis was observed in spindle-shaped mesenchymal cells, and inflammatory cell infiltration was obvious. At this stage, IMT was considered, and a recommendation was made that the IMT be completely removed by surgery.

On March 11, 2020, the patient underwent a right lower lobectomy under general anesthesia. A resected solid mass $(4.5 \mathrm{~cm} \times 4 \mathrm{~cm} \times 4 \mathrm{~cm})$ from a grayish-yellow lesion, $0.5 \mathrm{~cm}$ from the lung membrane, was sent for postoperative pathological examination. The pathological findings revealed a great deal of myofibroblasts, fibroblasts and collagen fibers in the lower right lung lesion, accompanied by a large number of plasma cells and foam cell infiltration, which were consistent with the features of IMT. No tumor cell was observed in the lower lobe stump region. No lymph node metastasis was found; however, plasma cell infiltration and necrotic nodules were observed in some lymph node samples. Immunohistochemical staining showed the positive expression of spinal muscular atrophy (SMA), cluster of differentiation (CD)38, CD68, Vimentin, and $\mathrm{Ki} 67$ (20\%), and the negative expression of anaplastic lymphoma kinase (ALK), desmin, and pan-cytokeratin (CK). After surgery, the patient recovered well and was discharged shortly thereafter. There was no recurrence during the postoperative 6-month follow up period. The patient's key blood indices at each time point are shown in Table 1; typical CT images and pathological photos are shown in Figure 1.

\section{Case 2}

A 49-year-old male patient was admitted to our hospital, complaining of an intermittent cough and abdominal pain, which he had experienced for 17 years. In the year preceding his admission, he had had a fever and facial swelling for 10 months. Some 10 months ago, the patient developed a fever without any known inducement. The fever usually appeared in the afternoon and night, and the patient's highest body temperature was $38^{\circ} \mathrm{C}$. The patient's right face was also swollen and had been painful since then. He had been diagnosed with pulmonary tuberculosis and hospitalized in Handan Central Hospital 6 months ago. However, he did not respond to the isoniazid $(\mathrm{H})$, rifampin (R), pyrazinamide $(\mathrm{Z})$, and ethambutol (E) (HRZE) anti-tuberculosis treatment. Some 4 months ago, he was admitted to The Fourth Hospital of Hebei Medical University and underwent a needle biopsy of the soft tissue near the right lateral pterygoid muscle. The pathological results still indicated inflammatory lesions, and tuberculosis was still considered. The continued application of HRZE tuberculosis treatment for 1 month did not relieve the patient's symptoms. Thus, the treatment was changed to isoniazid $(\mathrm{H})$, levofloxacin $(\mathrm{L})$, pyrazinamide (Z), and ethambutol (E) (HLZE). The patient had also undergone left lung fibro-blastoma surgery 6 years ago. 
Table 1 Blood indices at several key time points for Case 1

\begin{tabular}{|c|c|c|}
\hline Time & Indices & Value \\
\hline \multirow[t]{16}{*}{$2020-02-19$} & WBC $\left(10^{9}\right)$ & 10.18 \\
\hline & Neutrophils $\left(10^{9}\right)$ & 6.77 \\
\hline & Lymphocytes $\left(10^{9}\right)$ & 2.22 \\
\hline & Monocyte $\left(10^{9}\right)$ & 0.41 \\
\hline & $\operatorname{RBC}\left(10^{9}\right)$ & 4.8 \\
\hline & Hemoglobin (g/L) & 137 \\
\hline & Platelets $\left(10^{9}\right)$ & 304 \\
\hline & ALT (U/L) & 41 \\
\hline & AST (U/L) & 24 \\
\hline & ALP (U/L) & 93 \\
\hline & TBIL (U/L) & 5.7 \\
\hline & D-dimer value (mg/L) & 0.12 \\
\hline & CYFRA21-1 (ng/mL) & 1.8 \\
\hline & CEA (ng/mL) & 2.38 \\
\hline & $\operatorname{SCC}(n g / m L)$ & 2.12 \\
\hline & NSE (ng/mL) & 10.5 \\
\hline \multirow[t]{12}{*}{ 2020-03-09 } & WBC $\left(10^{9}\right)$ & 9.41 \\
\hline & Neutrophils $\left(10^{9}\right)$ & 6.61 \\
\hline & Lymphocytes $\left(10^{9}\right)$ & 2.03 \\
\hline & Monocyte $\left(10^{9}\right)$ & 0.3 \\
\hline & $\operatorname{RBC}\left(10^{9}\right)$ & 4.9 \\
\hline & Hemoglobin (g/L) & 141 \\
\hline & Platelets $\left(10^{9}\right)$ & 321 \\
\hline & ALT (U/L) & 46 \\
\hline & AST (U/L) & 29 \\
\hline & ALP (U/L) & 114 \\
\hline & TBIL (U/L) & 5.1 \\
\hline & D-dimer value (mg/L) & 0.16 \\
\hline
\end{tabular}

Table 1 (continued)

Some 17 years ago, the patient had been diagnosed with pulmonary tuberculosis and intestinal tuberculosis. $\mathrm{He}$ underwent surgical treatment for the intestinal tuberculosis. After surgery, he was given anti-tuberculosis drugs (e.g., streptomycin) for about 1 year. The drugs were stopped after his symptoms improved. There was no history of
Table 1 (continued)

\begin{tabular}{lll}
\hline Time & Indices & Value \\
\hline 2020-03-16 & WBC $\left(10^{9}\right)$ & 8.91 \\
& Neutrophils $\left(10^{9}\right)$ & 5.76 \\
& Lymphocytes $\left(10^{9}\right)$ & 2.22 \\
& Monocyte $\left(10^{9}\right)$ & 0.43 \\
& RBC $\left(10^{9}\right)$ & 4.19 \\
& Hemoglobin $(\mathrm{g} / \mathrm{L})$ & 119 \\
& Platelets $\left(10^{9}\right)$ & 307 \\
\hline
\end{tabular}

WBC, white blood cell; RBC, red blood cell; ALT, alanine aminotransferase; AST, aspartate aminotransferase; ALP, alkaline phosphatase; TBIL, total bilirubin; CYFRA21-1, cytokeratin 19 fragment; CEA, carcinoembryonic antigen; SCC, squamous cell carcinoma; NSE, neuron-specific enolase.

trauma or significant recent weight change. His mother and two children were alive and well. His father died of coronary heart disease (CHD), not thought to be hereditary.

After admission, the sputum culture results and analysis of the alveolar lavage fluid showed no fungal growth and tubercle bacillus; however, positive results for Streptococcus viridans were detected. On June 8, 2018, the CT imaging results showed that: (I) the left thorax was narrower than the opposite side, and the volume of the left lung was reduced; (II) the bilateral pleura were locally thickened and adhered; (III) a fluid density shadow in the right thoracic cavity; (IV) a linear dense shadow in the left upper lobe trachea; (V) multiple subpleural cord signs in the left lung; (VI) soft tissue mass shadows at the apex of the right upper lobe [maximum diameter $3.2 \mathrm{~cm}, 21$ Hounsfield unit (HU)], around which there was a thickened interlobular interval; and (VII) scattered patches and nodules in the upper right lung. On the same day, we punctured the upper lobe of the right lung and acquired a tissue sample. The pathological findings of the sample showed acute and chronic inflammation, accompanied by fibrosis. Additionally, foam cell aggregation and organization were observed in the alveoli.

The patient underwent surgical resection of the right upper lobe on June 15, 2018. After an incision of the parietal area of the right lung, a rough gray area could be touched and was resected (the area was $3.5 \mathrm{~cm} \times 3 \mathrm{~cm} \times$ $1.5 \mathrm{~cm}$, solid and flexible). As expected, the postoperative pathological analysis showed significant proliferation of fibrous tissue and fibroblasts in the upper lobe of the right lung, inflammatory cell infiltration, alveolar epithelial cell 

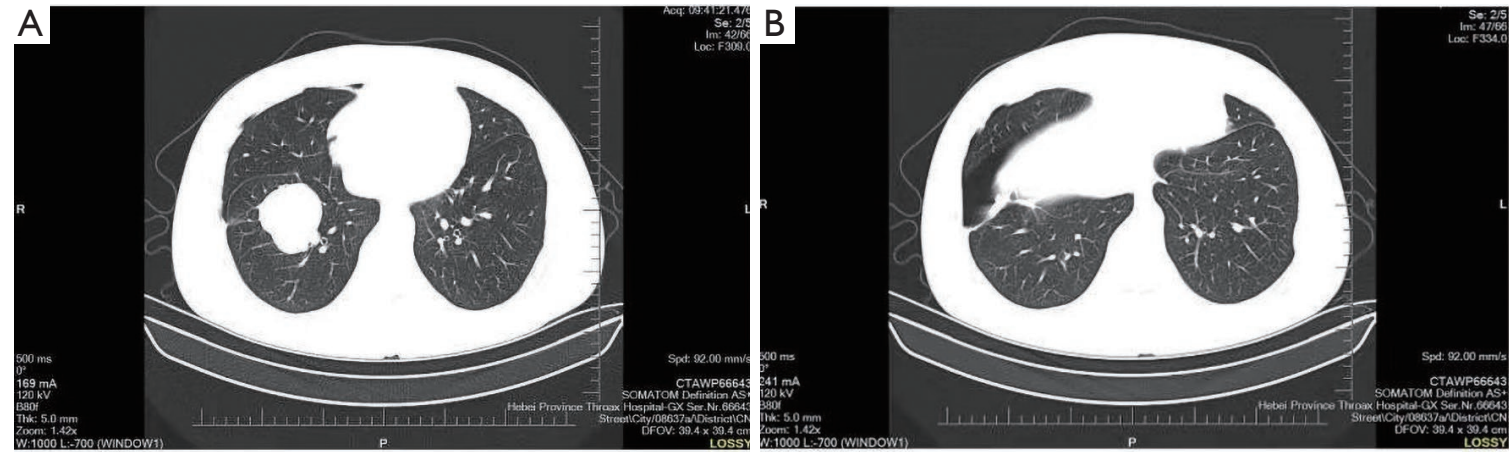

Figure 1 Typical CT images of Case 1 on February 13, 2020. (A) The upper lung; (B) the lower lung. CT, computed tomography.

proliferation, and phagocytes and foam cells in the alveolar cavity. Additionally, there were hemosiderin deposition and fibrosis, accompanied with organizing nodule formation. The immunohistochemical staining showed the positive expression of thyroid transcription factor 1 (TTF-1), CD68, Vimentin, Napsin A, and SMA. IMT was then considered. A soft gray tissue $(1.5 \mathrm{~cm} \times 0.8 \mathrm{~cm} \times 0.5 \mathrm{~cm})$ of the right upper lobe nodule was also removed. The pathological results showed organizing pneumonia-like changes, chronic inflammation, and foam cell aggregation. The patient's key blood indices at each time point are shown in Table 2; typical CT images and pathological photos are shown in Figure 2.

\section{Discussion}

IMT can occur in patients of all ages, but the average age of onset is 10 years old. For adult patients, it occurs more often in females, and is extremely rare in male adults. Due to its rarity and the scarce literature pertaining to its occurrence, it is often misdiagnosed in adult males. Our two male patients both experienced intermittent symptoms, but pulmonary IMTs were not suspected for a long time. Both patients were diagnosed with pulmonary tuberculosis before IMT was confirmed. To our knowledge, these patients are among the first pulmonary IMT cases. Both patients were adult males with lesions in the right lobe, a history of pulmonary tuberculosis, and a long period of refractory intermittent pulmonary inflammation.

Pulmonary IMT is known to be associated with chest pain and dyspnea, but patients with IMT may also be asymptomatic (1). Our cases showed that a main symptom of IMT was an intermittent cough; however, chest pain and dyspnea were not observed. Our findings suggest that a refractory cough in patients with pulmonary tuberculosis can be associated with the development of IMT. Interestingly, a fever accompanied by facial swelling may also be a potential symptom of IMT. Notably, a high fever was also reported to be an initial symptom of primary gastric IMT in an adult female patient (5). However, more evidence needs to be gathered to consolidate this association.

We need to carefully identified the pulmonary inflammatory in IMT and pneumonia. Their symptoms are remarkably similar, such as cough and fever. The main imaging features of an IMT include mass-like lesions, which may show lobular signs, pleural depression, mediastinal lymphadenopathy, and segmental atelectasis. Some cases may display a small amount of pleural effusion. However, there are very few calcification cases. Thus, it is difficult to diagnose IMT from imaging alone, and IMT is usually confirmed very late. Indeed, it is often not confirmed until a lung biopsy is required for pathological analysis. We suggest that the gold standard for the diagnosis of IMT is biopsy pathological composed of myofibroblastic spindle cells, accompanied by an inflammatory infiltrate of chronic inflammatory cells, such as fibroblasts, plasma cells, lymphocytes, or/and eosinophil. Occasionally, the amount of the puncture tissue may be small, and a diagnosis cannot then be confirmed until surgical treatment. So the pathological findings is still the most striking distinction between the pulmonary inflammatory in IMT and pneumonia as well as other lung cancer. In Case 1, a $5.6-\mathrm{cm}$ mass in the lower lobe of the right lung with a smooth edge and clear tumor-lung interface did not present any specific IMT imaging features. In Case 2, we observed multiple subpleural cord signs in the left lung, soft tissue mass shadows at the apex of the right upper lobe, a thickened interlobular interval, and scattered patches and nodules 
Table 2 Blood indices at several key time points for Case 2

\begin{tabular}{|c|c|c|}
\hline Time & Indices & Value \\
\hline \multirow[t]{19}{*}{ 2018-04-27 } & WBC $\left(10^{9}\right)$ & 7.14 \\
\hline & Neutrophils $\left(10^{9}\right)$ & 5.33 \\
\hline & Lymphocytes $\left(10^{9}\right)$ & 1.02 \\
\hline & Monocyte $\left(10^{9}\right)$ & 0.73 \\
\hline & $\operatorname{RBC}\left(10^{9}\right)$ & 3.94 \\
\hline & Hemoglobin (g/L) & 109 \\
\hline & Platelets $\left(10^{9}\right)$ & 267 \\
\hline & ALT (U/L) & 10 \\
\hline & AST (U/L) & 14 \\
\hline & ALP (U/L) & 82 \\
\hline & TBIL (U/L) & 7.2 \\
\hline & CYFRA21-1 (ng/mL) & 1.6 \\
\hline & CEA (ng/mL) & $<0.2$ \\
\hline & $\operatorname{AFP}(n g / m L)$ & 1.32 \\
\hline & CA125 (ng/mL) & 12.49 \\
\hline & CA153 (ng/mL) & 10.36 \\
\hline & CA199 (ng/mL) & 5.1 \\
\hline & $\mathrm{SCC}(\mathrm{ng} / \mathrm{mL})$ & 0.5 \\
\hline & NSE (ng/mL) & 7.34 \\
\hline \multirow[t]{11}{*}{$2020-05-16$} & WBC $\left(10^{9}\right)$ & 4.15 \\
\hline & Neutrophils $\left(10^{9}\right)$ & 2.58 \\
\hline & Lymphocytes $\left(10^{9}\right)$ & 1.21 \\
\hline & Monocyte $\left(10^{9}\right)$ & 0.28 \\
\hline & $\operatorname{RBC}\left(10^{9}\right)$ & 3.53 \\
\hline & Hemoglobin (g/L) & 96 \\
\hline & Platelets $\left(10^{9}\right)$ & 209 \\
\hline & ALT (U/L) & 8 \\
\hline & AST (U/L) & 16 \\
\hline & ALP (U/L) & 82 \\
\hline & TBIL (U/L) & 2.8 \\
\hline
\end{tabular}

Table 2 (continued)

in the upper right lung. These features are novel in the identification of IMTs. However, the diagnostic role of symptoms remains limited. A correct histological diagnosis is of the utmost importance, as IMTs closely resemble
Table 2 (continued)

\begin{tabular}{|c|c|c|}
\hline Time & Indices & Value \\
\hline \multirow[t]{11}{*}{ 2020-06-05 } & WBC $\left(10^{9}\right)$ & 5.09 \\
\hline & Neutrophils $\left(10^{9}\right)$ & 3.5 \\
\hline & Lymphocytes $\left(10^{9}\right)$ & 1.22 \\
\hline & Monocyte $\left(10^{9}\right)$ & 0.32 \\
\hline & $\operatorname{RBC}\left(10^{9}\right)$ & 3.69 \\
\hline & Hemoglobin (g/L) & 99 \\
\hline & Platelets $\left(10^{9}\right)$ & 244 \\
\hline & ALT (U/L) & 7 \\
\hline & AST (U/L) & 17 \\
\hline & ALP (U/L) & 79 \\
\hline & TBIL (U/L) & 3.8 \\
\hline \multirow[t]{11}{*}{$2020-06-23$} & WBC $\left(10^{9}\right)$ & 8.49 \\
\hline & Neutrophils $\left(10^{9}\right)$ & 5.66 \\
\hline & Lymphocytes $\left(10^{9}\right)$ & 1.62 \\
\hline & Monocyte $\left(10^{9}\right)$ & 0.55 \\
\hline & $\operatorname{RBC}\left(10^{9}\right)$ & 3.52 \\
\hline & Hemoglobin (g/L) & 97 \\
\hline & Platelets $\left(10^{9}\right)$ & 440 \\
\hline & ALT (U/L) & 9 \\
\hline & AST (U/L) & 13 \\
\hline & ALP (U/L) & 68 \\
\hline & TBIL (U/L) & 4.3 \\
\hline
\end{tabular}

WBC, white blood cell; RBC, red blood cell; ALT, alanine aminotransferase; AST, aspartate aminotransferase; ALP, alkaline phosphatase; TBIL, total bilirubin; CYFRA21-1, cytokeratin 19 fragment; CEA, carcinoembryonic antigen; AFP, alpha-fetoprotein; CA125, cancer antigen 125; CA153, cancer antigen 153; CA199, cancer antigen 199; SCC, squamous cell carcinoma; NSE, neuron-specific enolase.

other benign and malignant spindle cell tumors with myofibroblasts predominance (6).

In pathological analyses, IMT lesions have a distinctive histological appearance and usually follow a benign clinical course. Traditionally, IMT has been referred to by different names, including plasma cell granuloma, plasma cell pseudotumor, inflammatory myofibrohistiocytic proliferation, omental-mesenteric myxoid hamartoma, and inflammatory pseudotumor. Scholars have frequently 

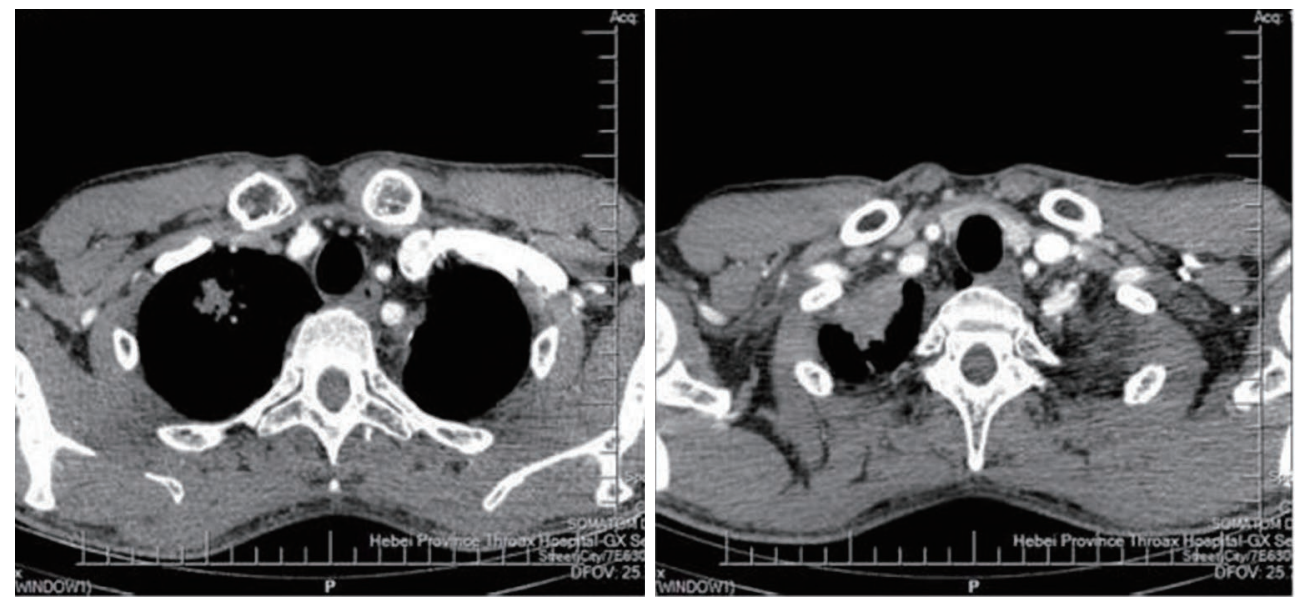

Figure 2 Typical CT images of Case 2 on June 8, 2018. CT, computed tomography.

confused inflammatory pseudo-tumors and IMTs. Inflammatory pseudotumor is a kind of immune reactivity disease which pathogenesis is unclear, organization pathology including pleomorphic inflammatory cells, lymphocytes, plasma cells, and eosinophils) and fiber tissue reaction vessels inflammation. According to the histological changes of idiopathic tumor samples, the disease can be divided into the lymphocyte infiltration type, hyperplasia of fibre type and mixed type. An IMT lesion was first described in a lung specimen in 1939, and it has been classified as a soft tissue tumor since 2002 (9). Its histopathological features usually include a mixture spectrum of spindle cells showing fibroblastic or myofibroblastic differentiation arrayed in fascicles or with storiform architecture. Varying inflammatory infiltrates, including plasma cells (prominent in many cases), lymphocytes, and histiocytes, are often admixed with the spindle proliferation and often obscure it (9). In addition to the IMT characteristics, the abovementioned histological features were consistently present in our two cases. Histopathology is particularly important in the diagnosis of IMT. However, given its scarcity, a combined molecular pathology analysis [such as an analysis of $A L K$ rearrangement, c-ros oncogene 1 (ROS1) rearrangement, and neurotrophic receptor tyrosine kinase 3 (NTRK3) mutation] may be more meaningful $(1,6)$. Generally, if the lesion is small, a local resection of the lung can be recommended. Alternately, a lobectomy and mediastinal lymph node dissection should be performed. In our two cases, surgical resections of each patient's right upper lobe was smoothly performed, and no recurrence was found. Further follow-up observation is still needed.

\section{Conclusions}

This article presented two rare cases of pulmonary IMTs in adult male patients. The patients presented with exceedingly different symptoms, CT imaging features, and pathological results from those reported in traditional records. These findings provide some novel references that will extend understandings of this rare disease.

\section{Acknowledgments}

Funding: None.

\section{Footnote}

Reporting Checklist: The authors have completed the CARE reporting checklist. Available at https://dx.doi. org/10.21037/tcr-21-1683

Conflicts of Interest: All authors have completed the ICMJE uniform disclosure form (available at https://dx.doi. org/10.21037/tcr-21-1683). The authors have no conflicts of interest to declare.

Ethical Statement: The authors are accountable for all aspects of the work in ensuring that questions related to the accuracy or integrity of any part of the work are appropriately investigated and resolved. All procedures performed in studies involving human participants were in accordance with the ethical standards of the institutional and/or national research committee(s) and with the Helsinki 
Declaration (as revised in 2013). Written informed consent was obtained from the patient for publication of this case report and accompanying images. A copy of the written consent is available for review by the editorial office of this journal.

Open Access Statement: This is an Open Access article distributed in accordance with the Creative Commons Attribution-NonCommercial-NoDerivs 4.0 International License (CC BY-NC-ND 4.0), which permits the noncommercial replication and distribution of the article with the strict proviso that no changes or edits are made and the original work is properly cited (including links to both the formal publication through the relevant DOI and the license). See: https://creativecommons.org/licenses/by-nc-nd/4.0/.

\section{References}

1. Bothale KA, Mahore SD, Patrikar AD, et al. A rare case of inflammatory myofibroblastoma of diaphragm. Indian J Surg 2013;75:243-6.

2. Coffin CM, Humphrey PA, Dehner LP. Extrapulmonary inflammatory myofibroblastic tumor: a clinical and pathological survey. Semin Diagn Pathol 1998;15:85-101.

3. Coffin CM, Watterson J, Priest JR, et al.

Cite this article as: Li H, He Y, Wang B, Song X, Zhao D, Yu H, Qi K, Liu T. Rare cases of pulmonary inflammatory myofibroblastic tumors in adult male patients: a case report. Transl Cancer Res 2021;10(9):4274-4280. doi: 10.21037/tcr21-1683
Extrapulmonary inflammatory myofibroblastic tumor (inflammatory pseudotumor). A clinicopathologic and immunohistochemical study of 84 cases. Am J Surg Pathol 1995;19:859-72.

4. Alloni R, Ancona G, Gallo I, et al. Melena as presentation of primary small intestine inflammatory myofibroblastic tumor in an adult woman. A case report 2015;86:S2239253X15024032.

5. Qiu JF, Shi YJ, Fang L, et al. High fever as an initial symptom of primary gastric inflammatory myofibroblastic tumor in an adult woman. Int J Clin Exp Med 2014;7:1468-73.

6. Gupta S, Goyal P, Yang Y, et al. Tracheal inflammatory myofibroblastoma: a rare tumor of the trachea. Cureus 2019;11:e4484.

7. Sziklavari Z, Droste A, Neu R, et al. Surgical treatment of pseudotumours of the lung. Zentralbl Chir 2018;143:90-5.

8. Kubal C, Ghotkar S, Gosney J, et al. Pleural inflammatory myofibroblastoma: a locally aggressive intra-thoracic tumour. J Cardiothorac Surg 2007;2:29.

9. Inamdar AA, Pulinthanathu R. Malignant transformation of inflammatory myofibroblastic tumor of urinary bladder: a rare case scenario. Bladder (San Franc) 2019;6:e39.

(English Language Editor: L. Huleatt) 